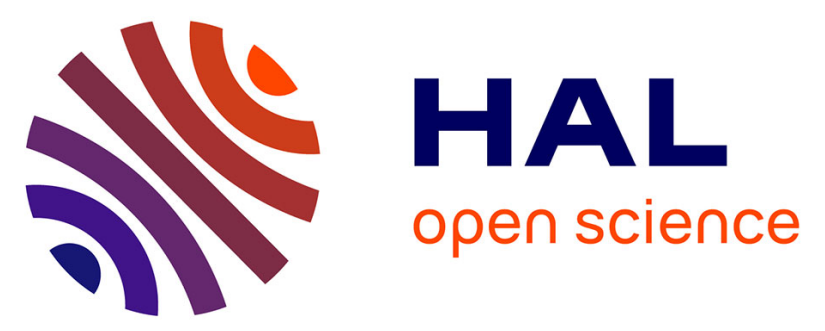

\title{
Numerical analysis of the coupling between the flow kinematics and the fiber orientation in eulerian simulations of dilute short fibers suspensions flows
}

Francisco Chinesta, Arnaud Poitou

\section{- To cite this version:}

Francisco Chinesta, Arnaud Poitou. Numerical analysis of the coupling between the flow kinematics and the fiber orientation in eulerian simulations of dilute short fibers suspensions flows. Canadian Journal of Chemical Engineering, 2003, 80 (6), pp.1107 - 1114. 10.1002/cjce.5450800612 . hal01007121

\section{HAL Id: hal-01007121 \\ https://hal.science/hal-01007121}

Submitted on 19 Feb 2017

HAL is a multi-disciplinary open access archive for the deposit and dissemination of scientific research documents, whether they are published or not. The documents may come from teaching and research institutions in France or abroad, or from public or private research centers.
L'archive ouverte pluridisciplinaire HAL, est destinée au dépôt et à la diffusion de documents scientifiques de niveau recherche, publiés ou non, émanant des établissements d'enseignement et de recherche français ou étrangers, des laboratoires publics ou privés. 


\title{
Numerical Analysis of the Coupling Between the Flow Kinematics and the Fiber Orientation in Eulerian Simulations of Dilute Short Fiber Suspensions Flows
}

\author{
Francisco Chinesta ${ }^{1}$ and Arnaud Poitou ${ }^{2}$
}

\author{
${ }^{1}$ LMSP (Laboratoire de Mécanique des Systèmes et des Procédés) CNRS-ENSAM-ESEM, 151 Boulevard de \\ l'Hôpital, F-75013 Paris, France and LRTMM-CNAM Paris, 292 rue Saint-Martin, F-75141 Paris cedex 03, France \\ ${ }^{2}$ LMT Cachan CNRS - ENS Cachan - Université Paris 6, 61 Avenue du President Wilson, F-94235 Cachan cedex, \\ France
}

$\mathrm{N}$ umerical modelling of non-Newtonian flows involves usually the coupling between equations of motion, which define an elliptical problem, and the fluid constitutive equation, which introduces an advection problem related to the fluid history. In short fiber suspensions (SFS) models, the extra-stress tensor depends on the fiber orientation whose evolution can be modeled from a transport problem. In all cases the flow kinematics and the fiber orientation are coupled: the kinematics of the flow governs the fiber orientation, and the presence and orientation of the fibers modify the flow kinematics. For example, in a contraction flow of a dilute suspension, large recirculating areas appear (Lipscomb et al., 1988).

If one uses SFS flows in material forming processes, the final fiber orientation state depends on the process (induced anisotropy). In this case we need to compute the coupled model (kinematics-fiber orientation) in order to predict the final mechanical properties of the conformed pieces (which depend strongly on the fiber orientation). Of major industrial interest is process optimization to align the fibers in structural pieces in the main stress directions.

The mechanical model governing the SFS flow is given by the following equations (Batchelor, 1970, 1971; Hand, 1962; Hinch and Leal, 1975, 1976; Meslin, 1999).

Firstly, the momentum balance equations, without inertia and mass terms:

$\operatorname{Div} \underline{\underline{0}}=\underline{0}$

where $g$ is the stress tensor.

Secondly, the incompressibility condition:

$\operatorname{Di} \underline{w}=\underline{0}$

where $\underline{v}$ represents the velocity field.
This paper focuses on an accurate evaluation of short fibers suspensions models coupling the flow kinematics with the fiber orientation evolution. In coupled models the flow kinematics is usually solved using the finite element method, where the fiber orientation is introduced in the constitutive equation through its value in some points (nodes or integration points). In this paper we will compare in a simple steady shear flow, the exact solutions of the extrastresses associated with the fibers' presence with the numerical simulations obtained using both the method of characteristics and the discontinuous Galerkin's method to solve the equation governing the generalized gradient evolution, in order to avoid the introduction of any closure relation. The error introduced if a quadratic closure relation is considered in the constitutive equation will be also quantified.

Ce travail porte sur l'analyse du couplage cinématique - orientation des fibres dans les modèles numériques des suspensions diluées de fibres courtes. Dans les modèles couplés, la cinématique est résolue avec une technique d'éléments finis, où l'orientation des fibres est considérée dans un certain nombre de points (les nœuds ou les points utilisés pour l'intégration numérique). Dans ce travail, nous comparons les extra-contraintes dans un écoulement de type cisaillement simple, obtenues numériquement avec la méthode des caractéristiques ainsi qu'avec une méthode d'éléments finis discontinus, avec la solution exacte connue pour ce type d'écoulement. Dans tous les cas, l'orientation a été calculée sans l'introduction de relations de fermeture. Ce fait nous permet également de quantifier la validité d'une fermeture quadratique pour le tenseur d'orientations.

Keywords: short fiber suspensions, transport equation, Fokker-Planck equation, quadratic closure relation. 
Thirdly, the constitutive equation for a dilute suspension of high aspect-ratio particles is given by:

$$
\underline{\underline{\sigma}}=-p \underline{\underline{I}}+2 \eta\left\{\underline{\underline{D}}+N_{p}(\underline{\underline{\underline{\underline{a}}}}: \underline{\underline{D}})\right\}
$$

where $p$ denotes the pressure, $\underline{I}$ the unit tensor, $\eta$ the viscosity (which depends on the chosen model as explained in Meslin, 1999), $D$ the strain rate tensor, $N_{p}$ a scalar parameter depending on both the fiber concentration and the fiber aspect ratio, ":" the double dyadic product, i.e. $[\underline{\underline{a}}: \underline{\underline{D}}]_{i j}=a_{i j k} D_{k l}$ and $\underline{\underline{\underline{a}}}$ the fourth-order orientation tensor defined by:

$\underline{\underline{\underline{a}}}=\int \underline{\rho} \otimes \underline{\rho} \otimes \underline{\rho} \otimes \underline{\rho} \Psi(\underline{\rho}) d \underline{\rho}$

where $\underline{\rho}$ is the unit vector aligned in the fiber axis direction, $\otimes$ denotes the dyadic product (i.e. $\left.(\underline{\rho} \otimes \underline{\rho})_{i j}=\rho_{i} \rho_{j}\right)$, and $\psi(\underline{\rho})$ is the orientation distribution function satisfying the normality condition:

$$
\int \Psi(\underline{\rho}) d \underline{\rho}=1
$$

If $\psi(\underline{\rho})=\delta(\underline{\rho}-\underline{\hat{\rho}})$, with $\delta()$ the Dirac's distribution, all the orientation probability is concentrated in the direction defined by $\hat{\rho}$, and the corresponding orientation tensor will be $\hat{a}=\hat{\rho} \otimes \hat{\rho} \otimes \hat{\rho} \otimes \hat{\rho}$.

We can also define the second order orientation tensor as:

$$
\underline{\underline{\underline{a}}}=\int \underline{\rho} \otimes \underline{\rho} \Psi(\underline{\rho}) d \underline{\rho}
$$

It is easy to verify that if $\psi(\underline{\rho})=\delta(\underline{\rho}-\underline{\rho})$, the fourth-order orientation tensor can be written as the tensorial product of the second order orientation tensor, i.e.:

$$
\underline{\underline{\underline{a}}}=\underline{\underline{a}} \otimes \underline{\underline{a}}
$$

For general expressions of $\psi(\rho)$ the previous relation is not exact, and in that case Equation (7) becomes a closure approximation known as quadratic closure relation. However, other closure relations are usually applied (Advani and Tucker, 1990).

In a planar case, the isotropic orientation state is defined by the uniform distribution function:

$$
\Psi(\underline{\rho})=\frac{1}{2 \pi}
$$

and then, the second order orientation tensor related to a planar isotropic orientation state is:

$$
\underline{\underline{a}}=\frac{\underline{I}}{2}
$$

From a physical point of view, we can consider that the eigenvalues of the second-order orientation tensor $(\underline{a})$ represent the probability of finding the fiber in the direction of the corresponding eigenvectors.

And lastly, if we consider spheroidal fibers, we can describe the orientation evolution with the Jeffery equation:

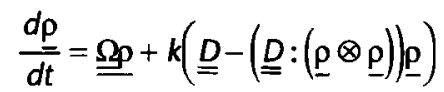

where $\Omega$ is the vorticity tensor, $k$ is a constant that depends on the fiber aspect ratio $r$ (fiber length to fiber diameter ratio):

$k=r^{2}-1 / r^{2}+1$

A solution of this equation is given by:

$\underline{\rho}=\frac{\underline{\underline{E}}_{k} \underline{\rho}_{0}}{\left\|\underline{\underline{E}} \underline{\rho}_{0}\right\|}$

where $\underline{\rho}_{0}$ is the initial orientation $\underline{\rho}_{0}=\underline{\rho}(t=0)$ and the transport $\underline{E}_{k}$ is the solution of the following transport equation:

$\frac{d \underline{\underline{\underline{E}}} k}{d t}=(\underline{\underline{\Omega}}+k \underline{\underline{D}}) \underline{\underline{E}}_{k}$

with $\underline{\underline{E}}_{k}(t=0)=\underline{l}$.

The evolution equation of the second-order orientation tensor results:

$\frac{d \underline{\underline{a}}}{d t}=\underline{\underline{\Omega} \underline{\underline{a}}}-\underline{\underline{a}} \underline{\underline{\underline{ }}}+k(\underline{\underline{D}} \underline{\underline{a}}+\underline{\underline{a}} \underline{\underline{D}}-2(\underline{\underline{\underline{a}}}: \underline{\underline{D}}))$

A similar equation can be derived for the evolution of the fourth-order orientation tensor, which in this case involves the sixth-order orientation tensor.

The solution of Equation (14) with a quadratic closure relation $(\underline{\underline{a}}=\underline{\underline{a}} \otimes \underline{\underline{a}})$ can be written as:

$\underline{\underline{a}}=\frac{\underline{\underline{E}}_{k} \underline{\underline{a}}_{0} \underline{\underline{E}}_{k}^{T}}{\operatorname{Tr}\left(\underline{\underline{E}}_{k} \underline{\underline{a}}_{0} \underline{\underline{E}}_{k}^{T}\right)}$

where $\underline{a}_{0}$ is the initial second order orientation tensor $\underline{\underline{a}}_{0}=\underline{a}(t=0)$.

Remark 1: Slender body theories (Batchelor, 1971; Dinh and Armstrong, 1984) assume that the fibers have an infinite aspect ratio, i.e. $k=1$ and the suspension viscosity is equal to the solvent viscosity. In that case, to describe the increase of the shear viscosity it is necessary to introduce diffusion effects to avoid the full alignment of the fibers with the flow (Folgar and Tucker, 1984). The orientation equation becomes:

$\frac{d \underline{\underline{\underline{a}}}}{d t}=\operatorname{Grad} \underline{\underline{v} \underline{\underline{a}}}+\underline{\underline{a}}(\operatorname{Crad} \underline{v})^{T}-2(\underline{\underline{\underline{a}}}: \underline{\underline{D}})+D_{r}\left(\underline{\underline{a}}-\frac{\underline{\underline{I}}}{2}\right)$ 
The introduction of the diffusion term $D_{r}(a-1 / 2)$ seems no longer necessary if we consider the spheroidal particles model because in that case the fact that $k \neq 1$ induces a continuous rotation of the fibers which can describe the rise in shear viscosity (Meslin, 1999).

In the numerical simulation of dilute fiber suspensions there are three kinds of numerical models:

1. Uncoupled models solve the kinematics without considering the presence of fibers. In this case, the equations of motion result in the Stokes's problem defined by Equations (1), (2) and (3) with $N_{p}=0$. From the velocity field obtained by the solution of the Stokes's problem, we can compute the fiber orientation evolution solving any of the models described previously. Givler et al. (1983) solved the Jeffery equation along the flow streamlines. To take into account different initial orientation distributions, a great number of fibers with different initial orientation are considered, and the averaging of their orientations at each point allows us to compute the different orientation tensors.

2. Coupled models using the Evans hypothesis solve the flow kinematics assuming that at each point of the fluid domain the fibers are aligned with the flow (local alignment hypothesis) (Lipscomb et al., 1988; Chiba et al., 1990). In this case, the flow model is given by Equations (1), (2) and (3), where the fiber orientation is given by:

$\underline{\rho}=\frac{\underline{v}}{\|\underline{v}\|}$

from which the expression for the second order orientation tensor $\underline{\underline{a}}$ results:

$\underline{\underline{a}}=\frac{\underline{v} \otimes \underline{v}}{\|\underline{\underline{v}}\|^{2}}$

which verifies exactly the quadratic closure relation $\underline{\underline{\underline{\underline{a}}}}=\underline{\underline{a}} \otimes \underline{\underline{a}}$.

3. Coupled models take into account both the dependence of the kinematics on the fiber orientation and the orientation induced by the flow kinematics. Usually the coupled models are solved by means of a fixed point strategy. In this case, at each iteration the flow kinematics results from Equations (1), (2) and (3) assuming the fiber orientation field at the previous iteration. From the kinematics just computed, the fiber orientation is updated solving the advection equation governing the evolution of the second- or the fourth-order orientation tensors, but in these cases the consideration of a closure relation is required. These advection equations can be integrated by using any accurate numerical technique for hyperbolic equations: the method of characteristics, SUPG or discontinuous finite element techniques, discontinuous finite volumes, ... (Rosenberg et al., 1990; Ausias, 1991; Altan et al., 1992; Souloumiac, 1996; Azaiez et al., 1997; Chiba and Nakamura, 1998; Poitou et al., 2000). Coupled models solving simultaneously the flow kinematics and the fiber orientation (fully coupled models) are rare in literature. The main difficulty of using fully coupled models is the different character of the model equations, which requires specific numerical techniques. Another particularity of this kind of model is the necessity of proceeding with a fully Eulerian description, whereas the fixed point coupled models can proceed with a Eulerian-Lagrangian mixed description, using, as previously described, the method of characteristics (the most accurate technique solving advection equations) in the solution of the orientation equation.

The first type of model does not allow prediction of the strong variations in the flow patterns that appear for example in contraction or expansion flows, even for dilute regimes (Lipscomb et al., 1988). The second type of model, extensively used, allows us to predict the existence of recirculating flow areas near the contraction corners (Lipscomb et al., 1988; Chiba et al., 1990). Moreover, as observed in several works (Towsend and Walters, 1993), and theoretically proven in Poitou et al. (2000) and Chinesta and Chaidron (2001), for fibers with infinite aspect ratio the local alignement of the fibers with the flow is the only solution in general recirculating flows. However, the local alignment cannot be applied either to divergent flows for which this solution is not stable, or to fibers with finite aspect ratio whose orientation changes continuously along their trajectory. As expected, the best modeling, widely used, consists of coupling flow kinematics and fiber orientation, as described for the third type of models. Different numerical strategies are used to solve the orientation equation: the method of characteristics, the SUPG or the discontinuous finite element methods or the discontinuous finite volumes method, using the second- or the fourth-order orientation tensors, as well as the generalized gradient $\underline{E}_{k}$ as variables. One of the main difficulties in using Eulerian discretisation techniques to compute the evolution of the tensorial orientation fields is the low accuracy of standard finite element interpolations of these orientation tensors, as discussed in Chinesta et al. (1999) and Chinesta et al. (2000).

On the other hand, the ellipticity of the equations of motion, when the orientation field is taken at the previous iteration, allows us to use a standard Galerkin formulation in finite elements. In general, this technique is used in most of the related works. Thus, in each element of the finite element mesh, the fiber orientation is considered only at some points (integration points). When the fibers are assumed of spheroidal shape and finite aspect ratio, it is well known that in a simple shear flow they cannot be fully aligned in the flow direction, rotating continuously. The higher the fiber aspect ratio the longer the fibers remain close to the flow direction, and faster is their rotation. We can expect that considering only the orientation state in some points we can predict an orientation state, which does not correspond with the physical reality. Thus, the probability of finding, at the integration points, the fibers aligned in the flow direction increases with the fibers' aspect ratio. In this case $N_{p} \underline{\underline{a}}: \underline{\underline{D}} \approx 0$ and the numerical model cannot predict the rise in she् ar viscosity experimentally observed. An alternative way to avoid this over-alignment through modeling is to consider the slender body theory, where, in spite of the fact that the fibers are assumed to have an infinite aspect ratio, the diffusion term introduced in Equation (16) avoids the full alignment of the fibers with the flow.

This paper evaluates the accuracy of coupled models using the spheroidal particles theory. We will analyse a simple shear flow, for which we can derive theoretically the rise in shear viscosity. These results will be compared with the different numerical simulations, without using any closure relation, in order to derive a conclusion about their accuracy. 
To take into account the effects of the fiber orientation on the flow kinematics, an accurate description of the fibers' orientation state in the equations of motion is required. This paper focuses on this area. The aim of this work is not the computation of coupled solutions, because, as commented previously, these calculations do not introduce additional difficulties if both an accurate strategy for solving advection equations and an accurate description of the orientation field into the equations of motion are performed. For this reason, only simple shear flows are considered in order to evaluate the capabilities of different numerical strategies to introduce accurately the fiber orientation distribution into the motion equations. In spite of the simplicity of such flows, the conclusions can be generalized to more general flows, where an effective coupling between the orientation and motion equations must be carried out for simulating industrial applications.

In the same way, other effects such as the fiber-fiber or wall-fiber interactions, which can be important in semi-concentrated or concentrated flows regimes, are not considered in this work, because to introduce accurately these effects more sophisticated mechanical models are required. In a first approximation, the introduction of diffusion effects in the equation governing the evolution of the fiber orientation distribution could describe the fiber-fiber interaction in semi-concentrated flows. In this case, the fiber orientation field is smoother than the one obtained without considering diffusion effects, and consequently, it can be accurately introduced into the equations of motion to compute coupled solutions.

Finally, this work concerns only planar orientation distributions. However, the analysis proposed in this paper can be applied to $3 \mathrm{D}$ orientation distributions as well as to general 3D flows.

\section{Orientation Solver}

In this section we will consider the velocity field as a datum. As just mentioned, we will use a numerical strategy to compute the fiber orientation field without the introduction of any closure relation. For this purpose, we propose to solve the evolution equation related to the generalized gradient $\underline{\underline{E}}_{k}$ :

$\frac{d \underline{\underline{\underline{E}}} k}{d t}=(\underline{\underline{\Omega}}+k \underline{\underline{D}}) \underline{\underline{E}}_{k}$

\section{A Lagrangian Discretisation: The Method of Characteristics}

To solve this linear advection equation (Equation 19) we consider the method of characteristics, which allows us to compute with very good accuracy the solution at each point. If we look for the solution of $\underline{E}_{k}$ at a point $x_{p}$, we need to reconstruct the trajectory, integrating upstream the equation of the characteristics:

$$
\frac{d t}{1}=-\frac{d \underline{x}}{\underline{v}} \text { with } \underline{x}(t=0)=\underline{x}_{P}
$$

until it reaches at the point $\underline{x}_{Q}$ the inflow boundary $\Gamma_{-}$of the fluid domain $\Omega$ :

$$
\Gamma_{-}=\{\underline{x} \in \partial \Omega \equiv \Gamma, \underline{v}(\underline{x}) \cdot \underline{n}(\underline{x})<0\}
$$

where $n$ is the unit outwards vector defined on the domain boundary $\partial \Omega$.

Now, we must integrate the orientation equation along the characteristics (streamlines for steady flows) from the boundary condition $\underline{E}_{k}\left(\underline{x}_{Q}\right)=\underline{E}_{k}\left(\underline{x} \in \Gamma_{-}\right)=\underline{\underline{l}}$ until we return to the starting point $x_{p} . \vec{A}$ fourth-order Runge-Kutta scheme with a control step has been used in the numerical integration, which leads to very accurate solutions.

The main limitation of this strategy is the necessity of reconstructing each trajectory associated with each point where the orientation solution must be computed. In general, this procedure is highly time consuming for the CPU. A semiLagrangian strategy that combines an integration by characteristics into each element and a Lagrangian interpolation on the mesh skeleton, reducing drastically the CPU time, was proposed by the authors in Chinesta et al. (2000). Another fully Eulerian strategy applies the discontinuous Galerkin method to solve Equation (19), as we will describe in the next section.

\section{A First-order Discontinuous Finite Element \\ Discretisation}

The simplest discontinuous Galerkin formulation lies in taking a value of $\underline{E}_{k}$ constant into each element of a finite element mesh. We will denote by $\underline{E}_{k}^{e}(t)$ the value of the generalized gradient $E_{k}$ in the element $\Omega^{e}$ at time $t$. The initial condition is given by $\underline{E}_{k}^{e}(t$ $=0)=1, \forall e \in\left[1, \ldots, N_{e}\right]$, where $N_{e}$ is the number of elements in

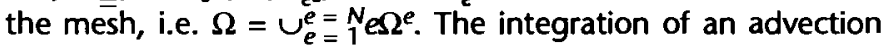
equation requires also a boundary condition on the inflow boundary $\Gamma_{-}$, which in our case results in $\underline{E}_{k}\left(x \in \Gamma_{-}\right)=\underline{\underline{l}}$.

The first-order time discretization is given by:

$\underline{\underline{E}}_{k}^{e}(t+\Delta t) \approx \underline{\underline{E}}_{k}^{e}(t)+\frac{\partial \underline{\underline{E}}_{k}^{e}}{\partial t} \Delta t \quad \forall e \in\left[1, \ldots, N_{e}\right]$

Now, a discontinuous Galerkin formulation is used to compute the time derivative. The integral conservation form, associated with the advection Equation (19), results in each element $\Omega^{e}$, taking into account the flow incompressibility $(\operatorname{Di} \underline{w}=0)$ ):

$$
\begin{aligned}
& \int_{\Omega^{e}} \frac{\partial \underline{\underline{\underline{E}}} \underline{k}^{e}}{\partial t} \partial \Omega=-\int_{\partial \Omega^{e}} \underline{\underline{E}}_{k}^{e} \underline{\underline{v}}^{T} \underline{n} \underline{\underline{n}}+\int_{\Omega^{e}}(\underline{\underline{\Omega}}+k \underline{\underline{D}}) \underline{\underline{E}}_{k}^{e} d \Omega \\
& \forall e \in\left[1, \ldots, N_{e}\right]
\end{aligned}
$$

where $\partial \Omega^{e}$ is the element boundary and $\underline{n}$ is the unit-outwards vector defined on the element boundary. As $E_{k}^{e}$ is not defined on the element boundaries, we take on the inflow boundary $\partial \Omega_{-}^{e}$, the existing value in the upstream element, whereas on the outflow boundary, $\partial \Omega_{+}^{e}$, we take the value existing in the element $\Omega^{e}$. Moreover, taking into account that $\underline{E}_{k}^{e}$ is constant into each element, we can finally write:

$$
\frac{\partial \underline{\underline{\underline{E}}}{ }_{k}^{e}}{\partial t}\left|\Omega^{e}\right|=\sum_{i}^{N_{u}^{e}} \underline{q}^{e_{i}^{-}} \underline{\underline{E}}_{k}^{e_{i}^{-}}-\underline{q}^{+} \underline{\underline{E}}_{k}^{e} \quad \forall e \in\left[1, \ldots, N_{e}\right]
$$

where $N_{u}^{e}$ is the number of upstream neighboring elements which are identified by the superscript $e_{i}, q^{F_{i}}$, is the flux coming 
from the upstream element $e_{i}$ and $q^{+}$is the flux leaving the element $\Omega^{e}$. Taking into account the incompressibility, we obtain:

$$
\sum_{i}^{N_{u}^{e}} \underline{q}^{e_{i}^{-}}=\underline{q}^{+} \quad \forall e \in\left[1, \ldots, N_{e}\right]
$$

The main advantage of this explicit strategy is a significant reduction in the CPU time.

\section{Computing the Fiber Orientation Tensors and the Rise in Shear Viscosity Computing the Orientation Tensors from the Generalized Gradient}

With the value of $\underline{E}_{k}$ computed at different points with the method of characteristics, or in each element using the justdescribed first-order discontinuous Galerkin method, the expression of the orientation tensors can be computed applying the numerical procedure described in this section. With the orientation distribution on the inflow boundary $\Psi_{0}\left(\underline{x} \in \Gamma_{-}, \underline{\rho}\right)$ done, the expression of $\underline{\underline{a}}$ and $\underline{\underline{\underline{a}}}$ can be obtained from their definition expressions:

$$
\begin{aligned}
& \underline{\underline{q}}(\underline{x})=\oint \underline{\rho} \otimes \underline{\underline{\rho}} \Psi(\underline{x}, \underline{\rho}) d \underline{\rho} \\
& \underline{\underline{a}}(\underline{x})=\oint \underline{\rho} \otimes \underline{\rho} \otimes \underline{\rho} \otimes \Psi(\underline{x}, \underline{\rho}) d \underline{\rho}
\end{aligned}
$$

If we denote by $\underline{\rho}_{\theta}$ the unit vector with components $\underline{\rho}_{\theta}^{T}=(\cos \theta$, $\sin \theta)$, we can then write:

$$
\underline{\underline{a}}(\underline{x})=\int_{0}^{2 \pi} \underline{\rho}_{\theta} \otimes \underline{p}_{\theta} \Psi(\underline{x}, \theta) d \theta
$$

and

$$
\underline{\underline{g}}(\underline{x})=\int_{0}^{2 \pi} \underline{\rho}_{\theta} \otimes \underline{\rho}_{\theta} \otimes \underline{\rho}_{\theta} \otimes \underline{\rho}_{\theta} \Psi(\underline{x}, \theta) d \theta
$$

We will be able to evaluate the previous expressions as soon as the fiber orientation distribution at any point $\underline{x}, \Psi(\underline{x}, \rho)$, is computed from the initial one, $\Psi_{0}$ (known on the inflow boundary), applying the following balance equation:

$$
\Psi_{0}\left(\underline{x}_{0}, \alpha\right) d \alpha=\Psi_{0}(\underline{x}, \theta) d \theta
$$

where $\alpha$ defines the fiber orientation at point $\underline{x}_{0}$, which, when the fiber reaches point $\underline{x}$ (both $\underline{x}_{0}$ and $\underline{x}$ are on the same trajectory), its orientation is defined by the angle $\theta$. Moreover, all the fibers whose initial orientation at point $\underline{x}_{0}$ is in the interval $[\alpha, \alpha$ $+d \alpha]$ will be in the oriented interval $[\theta, \theta+d \theta]$ when they reach the position $\underline{x}$.
To obtain $\alpha$ from $\theta$ we use the Jeffery equation:

$\underline{\rho}_{\alpha}=\frac{\underline{\underline{E}}_{K}^{-1}(\underline{x}) \underline{\rho}_{\theta}}{\left\|\underline{E}_{K}^{-1}(\underline{x}) \underline{\rho}_{\theta}\right\|}$

Now, if we consider $\theta+d \theta$, Equation (31) gives us the angle $\alpha^{*}$, from which we can obtain $d \alpha=\left|\alpha^{*}-\alpha\right|$.

Thus, we can compute for each angle $\theta, \Psi(\underline{x}, \theta) d \theta=\Psi_{0}\left(\underline{x}_{0}\right.$ $\alpha) d \alpha$, which allows us to obtain numerically the integrals defining both orientation tensors, Equations (28) and (29).

\section{Computing the Shear Viscosity}

Now, if we consider the constitutive equation, Equation (3), and the simple shear flow defined by $\underline{v}^{T}=(\dot{\gamma} y, 0)$, the shear viscosity $\eta_{s}$, defined by the ratio between the shear stress and the shear rate $\dot{\gamma}$, results:

$\eta_{s}=\frac{\sigma_{12}}{\dot{\gamma}}=\eta+2 N_{p} \eta a_{1212}=\eta+\Delta \eta$

where the symmetry relation $a_{1212}=a_{1212}$ has been introduced, and $\Delta \eta$ represents the rise in shear viscosity, which depends on $a_{1212}$

Remark 2: When the fibers have a quasi-infinite aspect ratio, $k=1$, the steady solution is given by the local alignment of the fiber with the flow, i.e. $\Psi(x, \theta)=\delta(x, \theta-0)$, from which it results $a_{1212}=0$, and consequently $\Delta \eta=0$.

Remark 3: When the fibers have a finite aspect ratio and the orientation distribution at each point of the fluid domain is given by the Dirac's function $\Psi(x, \theta)=\delta[x, \theta-\varphi(x)]$, then $a_{1212}$ $=a_{12}^{2}$, and consequently, the quadratic closure relation is exact.

\section{Numerical Examples}

If we consider the simple shear flow defined by the velocity field $\underline{v}^{T}=(\dot{\gamma} y, 0)$, with $\dot{\gamma}=1$ and the fibers with an aspect ratio such that $k=0.95$, and we focus our analysis on the orientation solution along the streamline defined by $y=1$, then the exact expression of $\underline{E}_{k}$, solution of Equation (19), results:

$$
\underline{E}_{k}(x)=\left(\begin{array}{cc}
\cos \left(\frac{\sqrt{1-k^{2}} x}{2}\right) & \frac{\sqrt{1-k^{2}}}{1-k} \sin \frac{\sqrt{1-k^{2}} x}{2} \\
\frac{\sqrt{1-k^{2}}}{1+k} \sin \frac{\sqrt{1-k^{2}} x}{2} & \cos \left(\frac{\sqrt{1-k^{2}} x}{2}\right)
\end{array}\right)
$$

From this equation we can come to the conclusion about the periodicity in the fibers' orientation. The spatial fiber orientation period $X$ is in our example $X=4 \pi / \sqrt{1-k^{2}}=40.24$.

We will consider the inflow boundary located at $x=0$, where in a first time, the fibers are aligned with the flow. Later, an isotropic fiber orientation on the inflow boundary will be considered.

Analysis of the Solution Along a Streamline for Fibers Aligned in the Flow Direction on the Inflow Boundary In this case, at each point $x$, the fibers will be perfectly aligned in the direction given by the Jeffery equation, i.e.: 
$\underline{\rho}(x)=\frac{\underline{\underline{E}}_{k}(x) \underline{\underline{\rho}}_{0}}{\left\|\underline{E}_{k}(x) \underline{\rho}_{0}\right\|} ; \underline{\rho}_{0}^{T}=(1,0)$

whose components are $\underline{\rho}(x)^{T}=\left(\rho_{1}(x), \rho_{2}(x)\right)$. So, $a_{1212}$ at point $x$ results:

$$
a_{1212}(x)=\rho_{1}^{2}(x) \rho_{2}^{2}(x)
$$

The average value in the spatial period $X$ can be considered, using the ergodic principle, as the exact steady local solution:

$$
\bar{a}_{1212}^{\text {exact }}=\frac{1}{x} \int_{0}^{x} \rho_{1}^{2}(x) \rho_{2}^{2}(x) d x
$$

that, in our case, results in $\bar{a}_{1212}^{\text {exact }}=0.059$.

Now, if we consider a uniform one-dimensional mesh on the interval $[0, X]$ including 20 elements, $N_{e}=20$, and we compute the values of $a_{1212}$ only at the center of gravity of each element, the mean value $\bar{a}_{1212}^{\text {num }}$ results:

$\bar{a}_{1212}^{\text {num }}=\frac{1}{N_{e}} \sum_{i=1}^{i=N_{e}} a_{1212}^{c d g, i}=0.073$

where $a_{1272}^{\text {cdq } i}$ denotes the value of $a_{1212}$ at the center of gravity of the element $\Omega^{i}$.

When the number of elements increases, the numerical solution $\bar{a}_{1212}^{\text {num }}$ converges to the exact one, i.e. $\bar{a}_{1212}^{\text {num }} \rightarrow \bar{a}_{1212}^{\text {exact }}=$ 0.059 (in our numerical example) when $N_{e} \rightarrow \infty$.

\section{Analysis of the Solution Along a Streamline for an Isotropic Fiber Orientation Distribution on the Inflow Boundary}

In this case, the variation of $a_{1212}$ is smoother than the previous one, because even if the fibers rotate very quickly, not all the fibers rotate at the same point, due to their different initial alignments. Thus, for the mesh considered in the previous example, we obtain:

$\bar{a}_{212}^{\text {num }}=\frac{1}{N_{e}} \sum_{i=1}^{N_{e}} a_{1212}^{c d g, i}=0.06$

which is very close to the exact value.

Now, we can evaluate $a_{12}$ at the center of gravity of each element $a_{12}^{\text {cdg, } i}$ as well as the mean value of $a_{1212}$ assuming a quadratic closure relation $\bar{a}_{1212}^{\text {num }}$ qcr.

$\bar{a}_{1212}^{\text {num, } q c r}=\frac{1}{N_{e}} \sum_{i=1}^{N_{e}}\left(a_{12}^{c d g, i}\right)^{2}=0.02$

which is far from the exact value.

Until now the generalized gradient $\underline{\underline{E}}_{k}$ has been obtained exactly from Equation (33) at the center of gravity of each element. For general flows, an accurate expression of $\underline{E}_{k}$ at a point requires the use of the method of characteristics, which, as we have previously indicated, is very expensive when considering CPU time. Moreover, we can expect that, by computing the average value of $\underline{E}_{k}$ in each element, using for example a discontinuous finite element method in the discretisation of Equation (19), an average of $a_{1212}$ will be obtained implicitly. In this situation we can define:

$\bar{a}_{1212}^{\text {num,d-fem }}=\frac{1}{N_{e}} \sum_{j=1}^{N_{e}} a_{1212}^{e}$

where $a_{1212}^{e}$ is the value found in the element $\Omega^{e}$, which is assumed to be constant into the element. The numerical application gives us the value $\bar{a}_{1212}^{\text {num }}=0.058$ very close again to the exact solution.

The main advantage of using a discontinuous finite element method to solve the equation governing the evolution of $\underline{E}_{k^{\prime}}$ Equation (19), is that it does not require the resolution of any linear system, and moreover, it reduces drastically the CPU time with respect to an integration by characteristics.

\section{Analysis of the Solution in the Whole Domain Using an Eulerian Discretisation}

In this section we consider the 2D flow model defined by the following kinematics:

$\underline{v}(x, y)=\left(\begin{array}{l}\dot{y} y \\ 0\end{array}\right),(x, y) \in[0, L] \times[0,1]$

If we take $\dot{\gamma}=1$, then the period $X$ of $\underline{E}_{k}$ depends on the $y$ coordinate in the following form:

$X(y)=\frac{4 \pi y}{\sqrt{1-k^{2}}}$

Now, if we define a uniform mesh in the fluid domain $[0, X(y=1)] \times[0,1]$, the characteristic element size near the upper wall, $y=1$, will be lower than the fiber orientation period $X(y)$, whereas in the area close to the lower boundary, $y=0$, the characteristic element size is greater than the fiber orientation period.

For this scenario, very close to actual general flow situations, we would like to compare results obtained by an accurate computation of tensor $\underline{E}_{k}$ at the center of gravity of the mesh elements (by means of the method of characteristics, for example) with the one obtained solving the evolution equation of $\underline{E}_{k}$, Equation (19), by means of the discontinuous Galerkin method (which assumes a value of $\underline{E}_{k}$ constant into each element, as described previously).

Using a mesh of 940 elements, $N_{e}=940$, and imposing the local alignment of the fibers with the flow on the inflow boundary, i.e. $\underline{\rho}_{0}^{T}=(1,0)$, we obtain the following results: 
$\bar{a}_{1212}^{\text {num }}=\frac{1}{N_{e}} \sum_{i=1}^{N_{e}} a_{1212}^{c d g, i}=0.060$

$$
\bar{a}_{1212}^{\text {num,d-fem }}=\frac{1}{N_{e}} \sum_{i=1}^{N_{e}} a_{1212}^{e}=0.055
$$

Now, if we assume on the inflow boundary an isotropic fiber orientation, i.e. $\Psi\left(x \in \Gamma_{-}\right)=1 / 2 \pi$ we obtain:

$$
\bar{a}_{1212}^{\text {num }}=\frac{1}{N_{e}} \sum_{i=1}^{N_{e}} a_{1212}^{c d g, i}=0.060
$$

$\bar{a}_{1212}^{\text {num,d-fem }}=\frac{1}{N_{e}} \sum_{i=1}^{N_{e}} a_{1212}^{e}=0.061$

From these results we can conclude that both techniques predict accurately the rise in shear viscosity. The solution accuracy is not significantly degraded when a considerable reduction in the number of degrees of freedom (in the order of 10 ) is performed. Even if the result obtained with the integration by characteristics seems slightly better, this technique requires much more CPU time than the second numerical technique. In order to improve the accuracy of the finite element technique, a local remeshing is needed. As in this technique we use a discontinuous approximation of the unknown field $\underline{E}_{k}$, we can proceed to non-structured local remeshings.

\section{Conclusions}

In this paper we have shown that in spite of the fast rotation of the fibers with finite aspect ratio in shear flows, the extrastresses are correctly evaluated in considering the fiber orientation only in a reduced number of points. If we use uniform meshes, the characteristic element length will be, in general, greater or smaller than the fiber rotation period. Even in that case, the solution accuracy is excellent, mainly when an isotropic fiber distribution is assumed on the inflow boundary. We have also noticed that very accurate solutions may be obtained by using the discontinuous Galerkin method, and they open new perspectives in the use of fully Eulerian coupled techniques. The highest deviations were found when the extrastress tensor was computed using a quadratic approximation for the fourth-order orientation tensor.

\section{Acknowledgements}

We want to thank Professor Claude Wolff, director of the research team at the Conservatoire National des Arts et Métiers (CNAM) of Paris, where a part of this work has been done.

\section{Nomenclature}

$\begin{array}{ll}\stackrel{a}{\underline{a}} & \text { second order orientation tensor, with components } a_{i j} \\ \underline{a}_{0} & \text { initial second order orientation tensor } \\ \underline{a} & \text { fourth order orientation tensor, with components } a_{i j k} \\ \stackrel{\bar{D}}{\underline{D}} & \text { strain rate tensor, with components } D_{i j}\left(s^{-1}\right) \\ D_{r} & \text { diffusion coefficient }\end{array}$
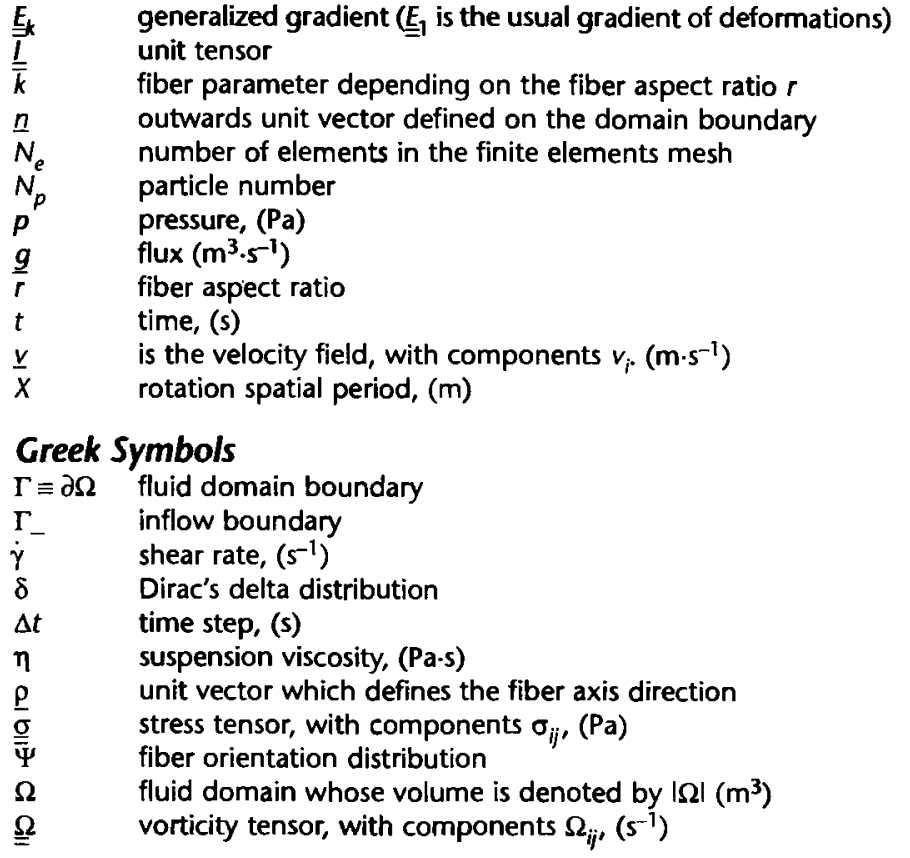

\section{Other Symbols}

$\otimes \quad$ dyadic product. Thus, the components of $\underline{u} \otimes \underline{v}$ are $u_{i} v_{i}$ : dyadic product, twice contracted

\section{References}

Advani, S.C. and C.L. Tucker III, "Closure Approximations for Threedimensional Structure Tensors", J. Rheol. 34, 367-386 (1990).

Altan, M.C., S.I. Guçeri, and R.B. Pipes, "Anisotropic Channel Flow of Fiber Suspensions", J. Non-Newt. Fluid Mech. 42, 65-83 (1992).

Ausias, G., "Étude de l'Extrusion de Tubes en Polymères Thermoplastiques Chargés de Fibres Courtes", PhD Thesis, ENSMP Paris, France (1991).

Azaiez, J., R. Guénette, and A. Ait-Kadi, "Investigation of the Abrupt Contraction Flow of Fiber Suspensions in Polymeric Fluids", J. NonNewtonian Fluid Mech. 73, 289-316 (1997).

Batchelor, G.K. "Slender-body Theory for Particles of Arbitrary Crosssection in Stokes Flow", I. Fluid Mech. 44, $419-440$ (1970).

Batchelor, G.K. "The Stress Generated in Non-Dilute Suspensions in Elongated Particles by Pure Straining Motion", J. Fluid Mech. 46, 813-829 (1971).

Chiba, K., K. Nakamura and D.V. Boger, "A Numerical Solution for the Flow of Dilute Fiber Suspensions Through an Axisymmetric Contraction", I. Non-Newtonian Fluid Mech. 35, 1-14 (1990).

Chiba, K. and K. Nakamura, "Numerical Solution of Fiber Suspensions Flow Through a Complex Channel", J. Non-Newtonian Fluid Mech. 78, 167-185 (1998).

Chinesta, F., A. Poitou and R. Torres, "Numerical Prediction of the Fiber Orientation in Steady Flows", Revue Européenne des Eléments Finis 8(4), 355-374 (1999).

Chinesta, F., A. Poitou and R. Torres, "A Semi-Lagrangian Strategy to Predict the Fiber Orientation in Steady Flows of Reinforced Thermoplastics", Comput. Methods Appl. Mech. Eng. 189, 233-247 (2000).

Chinesta, F. and G. Chaidron, "On the Steady Solution of Linear Advection Equations in Steady Recirculating Flows", J. NonNewtonian Fluid Mech. 98, 65-80 (2001).

Dinh, S.M. and R.C. Armstrong, "A Rheological Equation of State for Semi-Concentrated Fiber Suspensions", I Rheol. 28, 207-227 (1984).

Folgar, F. and C.L. Tucker III, "Orientation Behaviour of Fibers in Concentrated Suspensions", I. Reinf. Plast. Composites 3, 98-119 (1984).

Givler, C., M.J. Crochet and R.P. Pipes, "Numerical Prediction of Fiber Orientation in Dilute Suspensions", J. Compos. Mater. 17, 330-343 (1983). 
Hand, G.L., "A Theory of Anisotropic Fluids", J. Fluid Mech. 13, 33-46 (1962).

Hinch, E.J. and L.G. Leal, "Constitutive Equations in Suspension Mechanics. Part I", J. Fluid Mech. 71, 481-495 (1975).

Hinch, E.J. and L.G. Leal, "Constitutive Equations in Suspension Mechanics. Part II", J. Fluid Mech. 76, 187-208 (1976).

Lipscomb, G.G., M.M. Denn, D.U. Hur and D.V. Boger, "The Flow of Fiber Suspensions in Complex Geometries", J. Non-Newt. Fluid Mech. 26, 297-325 (1988).

Meslin, F. and A. Poitou, "Viscosité en Cisaillement d'un Composite Fibres Courtes à l'État Fondu", C.R. Acad. Sci. Paris 327, 559-565 (1999).

Poitou, A., F. Chinesta and R. Torres, "Numerical Simulation of the Steady Recirculating Flows of Fibers Suspensions", J. Non-Newtonian Fluid Mech. 90, 65-80 (2000).
Rosenberg, J., M.M. Denn and R. Keunings, "Simulation of NonRecirculating Flows of Dilute Fiber Suspensions", J. Non-Newtonian Fluid Mech. 37, 317-345 (1990).

Souloumiac, B. "Étude Rhéologique, Modélisation et Simulation Numérique de l'Écoulement des Thermoplastiques Chargés de Fibres Courtes", PhD Thesis, ENSMP, Paris, France (1996).

Townsend, P. and K. Walters, "Expansion Flows of Non-Newtonian Liquids", Chem. Eng. Sci. 49(5), 749-763 (1993). 